\title{
Chest X-rays in culture-negative pulmonary tuberculosis: early determination is superior to late determination
}

\author{
Yong-Soo Kwon
}

Department of Internal Medicine, Chonnam National University

Hospital, Gwangju, Korea

Received: August 6, 2020

Accepted: August 13, 2020

\section{Correspondence to}

Yong-Soo Kwon, M.D.

Department of Internal Medicine, Chonnam National University Hospital, 42 Jebong-ro, Dong-gu, Gwangju 61469, Korea Tel: $+82-62-220-6575$

Fax: $+82-62-225-8578$

E-mail:yskwon@jnu.ac.kr https://orcid.org/0000-00015121-4488

\section{See Article on Page 1145-1153}

Although tuberculosis (TB) is a curable disease, it represents a major worldwide health concern. Approximately 10 million TB cases and 1.45 million TB-related deaths were reported in 2018 [1]. Early detection and appropriate treatment improve both TB outcomes and control efforts. Sputum smear microscopy is a rapid, simple, and inexpensive tool for detecting active TB. However, it is associated with low and variable sensitivity (range, $32 \%$ to $94 \%$ ) [2]. The nucleic acid amplification test for TB is highly sensitive and specific, but has variable sensitivity and specificity [3]. Mycobacterial culture is a confirmatory tool for the diagnosis of active TB and is associated with a false-negative rate of $10 \%$, and requires 6 to 8 weeks [3]. Therefore, chest imaging studies are central for the diagnosis of TB, especially in cases of pulmonary TB where sputum mycobacterial presence cannot be confirmed. A thorough clinical and radiographic follow-up 2 to 3 months after treatment initiation is recommended for patients with pulmonary TB to eliminate the possibility of other diseas- es [4]; however, these changes do not provide strong evidence for the final diagnosis of pulmonary TB.

In this issue, Lee et al. [5] evaluated the clinical significance of chest $\mathrm{X}$-ray changes at different time points ( 1 or 2 months after treatment initiation) on the final pulmonary TB diagnosis in patients with negative culture test results. Patients suspected of having pulmonary TB $(\mathrm{n}=120)$ were enrolled in the study. Patients who failed to show improvement in the 1-month chest radiograph had more nontuberculous diseases in their final diagnosis than those who failed to show improvement in the 2-month chest radiograph $(34 / 65$ [52.3\%] vs. $20 / 50$ [33.8\%]). Therefore, the authors concluded that chest radiography 1-month after treatment initiation for TB was best for determining the final diagnosis in patients with suspected pulmonary TB.

These findings will aid decisions regarding continuation of TB treatment and help reduce unnecessary administration of TB drugs and adverse drug reactions in patients with nontuberculous diseases. Moreover, it may prevent the progression of other diseases. However, radiological changes after TB treatment are in- 
adequate for the final diagnosis of pulmonary TB in patients without bacteriological confirmation as well the effect of TB treatment on outcomes and relapses. The variability of radiological changes and presence of significant residual lesions after the completion of TB treatment contribute to this shortfall [6].

In South Korea, TB is a serious public health problem. Patients with chest radiographic findings that suggest active TB are generally diagnosed with pulmonary TB and treated with anti-TB drugs [7]. This approach can lead to improper diagnoses and inappropriate or unnecessary administration of therapeutics.

The most common nontuberculous diagnosis in this study was nontuberculous mycobacterial pulmonary disease (NTM-PD). An early differential diagnosis of NTM-PD from pulmonary TB is important because its therapeutic regimen differs from that of pulmonary $\mathrm{TB}$ and it does not require contact tracing [7]. However, a considerable amount of overlap exists between clinical and radiographic features of pulmonary TB and NTM-PD $[8,9]$. Therefore, radiographic findings alone cannot differentiate the two diseases and additional tests are required to obtain mycobacterial confirmation in clinical specimens. The recovery rate of NTM-PD from respiratory specimens and the number of patients with NTM-PD have increased in South Korea [10]. Therefore, rapid and accurate identification of Mycobacterium tuberculosis and its differentiation from the causative agent of NTM-PD in positive culture isolates is essential. Furthermore, correct identification of the NTM-PD causative agent is crucial because there are species-specific clinical features related to drug resistance, treatment regimen, and prognosis of disease [10]. Since symptoms, radiographic findings, and direct microscopy of respiratory specimens are insufficient to differentiate between pulmonary TB and NTM-PD, mycobacterial culturing and correct identification are the current gold standard [10].

Early TB treatment is crucial for effective prevention and treatment. However, misdiagnosis in patients without bacteriological confirmation is common. The observation of chest radiographic changes 1 month after treatment initiation may improve diagnosis in patients suspected of having pulmonary TB.

\section{Conflict of interest}

No potential conflict of interest relevant to this article was reported.

\section{REFERENCES}

1. World Health Organization. Global tuberculosis report 2019 [Internet]. Geneva (CH): WHO, 2019 [cited 2020 Aug 14]. Available from: https://apps.who.int/iris/bitstream/ handle/10665/329368/9789241565714-eng.pdf?ua=1.

2. Steingart KR, Henry M, Ng V, et al. Fluorescence versus conventional sputum smear microscopy for tuberculosis: a systematic review. Lancet Infect Dis 2006;6:570-581.

3. Lewinsohn DM, Leonard MK, LoBue PA, et al. Official American Thoracic Society/Infectious Diseases Society of America/Centers for Disease Control and Prevention clinical practice guidelines: diagnosis of tuberculosis in adults and children. Clin Infect Dis 2017;64:e1-e33.

4. Nahid P, Dorman SE, Alipanah N, et al. Official American Thoracic Society/Centers for Disease Control and Prevention/Infectious Diseases Society of America clinical practice guidelines: treatment of drug-susceptible tuberculosis. Clin Infect Dis 2016;63:e147-e195.

5. Lee JH, Kim OH, Kim YJ, Shim TS, Jo KW. Changes in chest X-ray findings in 1-and 2-month group after treatment initiation for suspected pulmonary tuberculosis. Korean J Intern Med 2020;35:1145-1153.

6. Seon HJ, Kim YI, Lim SC, Kim YH, Kwon YS. Clinical significance of residual lesions in chest computed tomography after anti-tuberculosis treatment. Int J Tuberc Lung Dis 2014;18:341-346.

7. Kwon YS, Koh WJ. Diagnosis of pulmonary tuberculosis and nontuberculous mycobacterial lung disease in Korea. Tuberc Respir Dis (Seoul) 2014;77:1-5.

8. Kendall BA, Varley CD, Choi D, et al. Distinguishing tuberculosis from nontuberculous mycobacteria lung disease, Oregon, USA. Emerg Infect Dis 2011;17:506-509.

9. Koh WJ, Yu CM, Suh GY, et al. Pulmonary TB and NTM lung disease: comparison of characteristics in patients with AFB smear-positive sputum. Int J Tuberc Lung Dis 2006;10:1001-1007.

10. Kwon YS, Koh WJ. Diagnosis and treatment of nontuberculous mycobacterial lung disease. J Korean Med Sci 2016;31:649-659. 